\section{Biopanning Phage Display Libraries Using Magnetic Beads Vs. Polystyrene Plates}

BioTechniques 26:208-214 (February 1999)

Phage display of random peptides (10) has developed into a very useful technology for the discovery of novel peptide ligands that bind to diverse targets (2). Selecting phage with desirable binding activities, or "biopanning", relies on the immobilization of a target protein on a solid support, which allows for the physical separation of binding from nonbinding phage virions. Historically, target molecules such as antibodies have been immobilized on plastic dishes either by passive adsorption or by biotinylation of the antibodies followed by their capture on streptavidincoated plates. Passive adsorption requires no chemical modification of the target molecule that might interfere with ligand-binding activity; however, target molecules immobilized by simple adsorption could orient in such a way as to obscure the binding pocket, thus inhibiting efficient ligand binding.

Paramagnetic beads have recently become widely used in a variety of molecular and cell biological applications. Such beads have been used $(i)$ to isolate single-stranded DNA for sequencing $(1,12)$ or polymerase chain reaction (PCR) (6), (ii) for purification of specific cell subsets from mixed populations $(5,8)$, (iii) for isolation of cDNA for library generation (9), (iv) for isolation of acrosome-reactive human sperm (4), (v) as supports for viral PCR products for sandwich hybridization assays (13) and (vi) for screening phage display libraries $(7,11)$.

There are a number of theoretical advantages to using paramagnetic beads as supports for immobilizing target molecules to biopan phage libraries. These include $(i)$ increased surface area for binding, (ii) increased ease and thoroughness of washing and (iii) in the case of antibody targets, the ability to appropriately orient the binding domains. In this study, we compared the efficiency of biopanning by traditional plate vs. magnetic bead methods using a monoclonal antibody (MAb) to human Interleukin (IL)-8. Use of the same target, phage library, incubation times, washing and elution conditions allowed rigorous comparison of the relative efficiencies of these two methods.

A commercial anti-human IL-8 MAb (HIL-8NR7; Devaron, Dayton, NJ, USA) was used to affinity-select binding virions from a phage library designated D38, which displays 38 random amino acids as an N-terminal fu- sion with the pIII protein of M13. The D38 library was constructed as described previously (3). Phage were subjected to both a plate and a magnetic bead biopanning procedure. Briefly, $100 \mu \mathrm{L}$ of anti-IgG-coated magnetic beads (Dynabeads ${ }^{\circledR}$ M-45 rat antimouse IgG1; Dynal, Lake Success, NY, USA) were added to a $15-\mathrm{mL}$ polypropylene centrifuge tube and "loaded" with anti-IL-8 by overnight incubation in $10 \mathrm{~mL}$ of antibody solu-

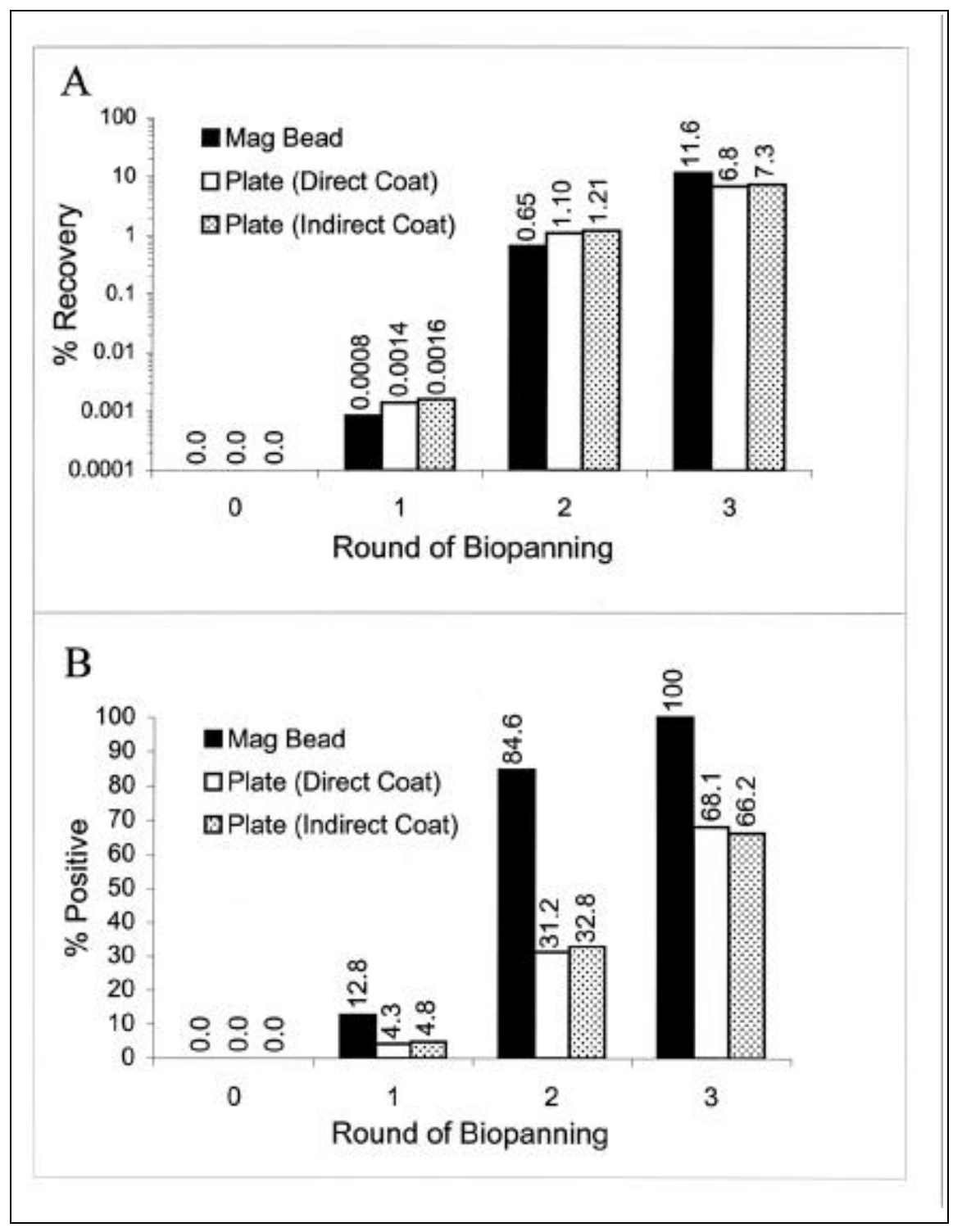

Figure 1. Comparison of magnetic bead vs. plate methods by plaque lift analysis. Percent recovery and percent positive values were obtained over three rounds of biopanning. A phage library (D38) was biopanned using the same target antibody (anti-human IL-8) using different immobilization methods: $(i)$ Mag bead, magnetic bead; (ii) Plate (Direct Coat), polystyrene plate direct antibody coating or (iii) Plate (Indirect Coat), indirect antibody coating. (A) Percent recoveries were determined by the following equation, where pfu is plaque-forming units: [(output pfu/input pfu) $\times 100]$. (B) Percent positives determined by plaque lift using the target antibody (see text). 


\section{Benchmarks}

tion, diluted to $1 \mu \mathrm{g} / \mathrm{mL}$ in SuperBlock ${ }^{\circledR}$ Blocking Buffer in TBS (TBS SuperBlock) (Pierce Chemical, Rockford, IL, USA) at $4^{\circ} \mathrm{C}$ with gentle rotation. Plates were coated by using either a "direct" or "indirect" coating method. For the direct method, 24-well Falcon ${ }^{\circledR}$ polystyrene plates (Catalog No. 1147; Becton-Dickinson Labware, Bedford, MA, USA) were coated with $1 \mathrm{~mL}$ of the same anti-IL- 8 antibody solution (1 $\mu \mathrm{g} / \mathrm{mL}$ in TBS SuperBlock) at $4^{\circ} \mathrm{C}$ overnight. For the indirect method, the same plates were first coated with exactly the same rat anti-mouse $\operatorname{IgG}$ used by Dynal to coat their magnetic beads (clone L0-MG1-13; Dynal) using the same conditions as the direct coating method above. After pre-coating with the rat anti-mouse-IgG capture antibody, the plates were then coated with the anti-IL-8 antibody as described for the direct coating method. Both beads and plates were then blocked at room temperature (RT) in TBS SuperBlock for $1 \mathrm{~h}$ with gentle shaking. The beads and plates were washed $3 \times$ in Trisbuffered saline plus $0.1 \%$ Tween ${ }^{\circledR} 20$ (TBST). The actual biopanning involved the addition of $1 \times 10^{10}$ plaqueforming units (pfu) from the D38 library in $1 \mathrm{~mL}$ of TBS SuperBlock to both the beads and the direct and indirect coated wells. The mixtures were rotated gently for $2 \mathrm{~h}$ at RT and then washed $10 \times$ in TBST. Binding phage were eluted by incubation in $300 \mu \mathrm{L}$ of $50 \mathrm{mM}$ glycine- $\mathrm{HCl}, \mathrm{pH} 2.2$, for 10 $\mathrm{min}$ at RT. Eluates were immediately neutralized with $8 \mu \mathrm{L}$ of $2 \mathrm{M}$ Tris- $\mathrm{HCl}$ and stored at $4^{\circ} \mathrm{C}$.

Binding phage were amplified by (i) adding the neutralized eluate to an equal volume of a fresh overnight culture of JS5 E. coli, (ii) diluting the mixture in $20 \mathrm{~mL}$ of $2 \times$ YT medium containing $12.0 \mu \mathrm{g} / \mathrm{mL}$ tetracycline and (iii) shaking overnight at $37^{\circ} \mathrm{C}$. Bacte- ria were pelleted for $5 \mathrm{~min}$ at $16000 \times \mathrm{g}$ in a microcentrifuge, and $100 \mu \mathrm{L}$ of the supernatant containing the amplified phage were used for subsequent rounds of biopanning. Biopanning was repeated for a total of 3 rounds for both magnetic beads and polystyrene plates.

All phage titering was done using JS5 E. coli and plated on $2 \times$ YT top agar and agar plates (Difco Laboratories, Detroit, MI, USA). The proportion of antibody-binding phage was determined by performing plaque lifts. Phage from each round of biopanning (magnetic bead and plate) were plated to a density of approximately 500 plaques per plate. Nitrocellulose filters were placed on the plates and incubated at $37^{\circ} \mathrm{C}$ for $1 \mathrm{~h}$. Filters were removed, washed briefly in TBST and blocked for $1 \mathrm{~h}$ at RT, followed by four additional washes in TBST. Filters were incubated for $1 \mathrm{~h}$ at RT in a solution of the anti-IL-8 antibody $(1 \mu \mathrm{g} / \mathrm{mL}$ in 


\section{Benchmarks}

TBS SuperBlock) and then rewashed $4 \times$ in TBST. Secondary antibody (goat antimouse alkaline phosphatase, Cata$\log$ No. 075-1802; Kirkegaard \& Perry, Gaithersburg, MD, USA) diluted 1:1000 in TBS SuperBlock was added to the filters and incubated for $1 \mathrm{~h}$ at RT. Filters were washed $4 \times$ in TBST and $2 \times$ in TBS and then developed using nitro blue tetrazolium/5-bromo-4chloro-3-indolyl (NBT/BCIP) developing reagent (Sigma Chemical, St. Louis, MO, USA) according to manufacturer's instructions. All titering and plaque lifts were performed in triplicate; the data reported represent arithmetic means. For all the data points, standard errors were less than $3 \%$.

Figure 1A shows that there is little difference in the total recovery of phage when comparing magnetic beads vs. both direct and indirect coated plates over three rounds of biopanning. However, the recovery of specific anti-IL-8 binding phage (as determined by plaque lift using the anti-IL-8 MAb), was significantly higher in the bead panning. Even after the first round of selection, there was a threefold increase in the number of positive plaques seen in the magnetic bead relative to both plate biopannings (Figure 1B). After the second round of biopanning, almost $85 \%$ of the magnetic bead-panned phage were positive; whereas, only $31.2 \%$ of the direct coated and $32.8 \%$ of the indirect coated plate-isolated phage were positive. By the third round of selection, the recoveries of plaque-positive IL-8 binding phage were $100 \%$ vs. $68.1 \%$ and $66.2 \%$ for the bead direct and indirect coated plate pannings, respectively. A total of 48 positive plaques were picked from both plate (direct and indirect) and magnetic bead biopanning methods. DNA was prepared from these phage clones and subjected to sequence analysis. All 144 positive clones sequenced from both biopanning methods contained the HPKF motif somewhere within the 38-residue sequence displayed. This motif sequence is also present in the original antigenic peptide fragment of human IL-8 against which the MAb was raised (human IL-8 residues 14-25; SKPFHPKFIKEL) and likely defines the specific epitope recognized by the MAb. This motif was found only in phage from plaques iden- tified as positive by plaque lift (none of 84 plaque lift-negative clones subjected to sequence analysis contained the HPKF sequence).

These results demonstrate that biopanning using magnetic beads is significantly more effective than plate biopanning for isolating specific binding phage. This finding has three practical consequences for investigators attempting to isolate phage capable of binding to biological targets. First, in this case, there is no significant difference between direct vs. indirect antibody-coating methods for plate biopanning. Second, in those cases in which actual binding phage are extremely rare in the parent library, it is crucial that these phage be recovered in the very first round of selection. Any technique that increases the efficiency of this initial capture will obviously be of particular value in these cases. Third, the earlier the round of selection in which binding phage are recognized, the more likely that the diversity of the recovered phage will be high. This can effectively minimize the repetitive isolation of "sibling" phage, i.e., identical phage sequences derived from replication of a single parent. Repetitive isolation of sibling phage wastes time and resources and could obscure the isolation of other phage with more valuable properties. The magnetic bead affinity selection procedure described here offers distinct advantages over traditional plastic dish biopanning in both respects. The increase observed in the efficiency of paramagnetic beads relative to plastic dishes as a target-immobilizing substrate would appear to make them the medium of choice for affinity screening of phage-display libraries.

\section{REFERENCES}

1.Alderton, R.P., L.M. Eccleston, R.P. Howe, C.A. Read, M.A. Reeve and S. Beck. 1992. Magnetic bead purification of M13 DNA sequencing templates. Anal. Biochem. 210:166169.

2.Katz, B.A. 1997. Structural and mechanistic determinants of affinity and specificity of ligands discovered or engineered by phage display. Ann. Rev. Biophys. Biomol. Struct. 26:27-45.

3.McConnell, S.J., A.J. Uveges, D.M. Fowlkes and D.G. Spinella. 1996. Construction and screening of M13 phage libraries displaying long random peptides. Mol. Diversity 1:165-
176.

4.Okabe, M., S. Matzno, M. Nagira, X. Ying, Y. Kohama and T. Mimura. 1992. Collection of acrosome-reacted human sperm using monoclonal antibody-coated paramagnetic beads. Mol. Reprod. Dev. 32:389-393.

5.Ossendorp, F.A., P.F. Bruning, J.A.M. Van den Brink and M. De Boer. 1989. Efficient selection of high-affinity B cell hybridomas using antigen-coated magnetic beads. J. Immunol. Methods 120:191-200.

6.Rudi, K., M. Kroken, O.J. Dahlberg, A. Deggerdal, K.S. Jakobsen and F. Larsen. 1997. Rapid, universal method to isolate PCRready DNA using magnetic beads. BioTechniques 22:506-511.

7.Sawyer, C., J. Embleton and C. Dean. 1997. Methodology for selection of human antibodies to membrane protein from a phage-display library. J. Immunol. Methods 204:193-203.

8.Siegel, D.L., T.Y. Chang, S.L. Russell and V.Y. Bunya. 1997. Isolation of cell surface human monoclonal antibodies using phage display and magnetically-activated cell sorting: applications in immunohematology. J. Immunol. Methods 206:73-85.

9.Shepard, A.R. and J.L. Rae. 1997. Magnetic bead capture of cDNAs from double-stranded plasmid cDNA libraries. Nucleic Acids Res. 25:3183-3185.

10.Smith, G.P. 1985. Filamentous fusion phage: novel expression vectors that display cloned antigens on the virion surface. Science 228 : 1315-1317.

11.Wang, L., M.Z. Radic, D. Seigel, T. Chang, J. Bracy and U. Galili. 1997. Cloning of antiGal Fabs from combinatorial phage display libraries: structural analysis and comparison of $\mathrm{Fab}$ expression in $\mathrm{pComb} 3 \mathrm{H}$ and $\mathrm{pComb} 8$ phage. Mol. Immunol. 34:609-618.

12.Wang, S., M. Krinks and M. Moos, Jr. 1995. DNA sequencing from single phage plaques using solid-phase magnetic capture. BioTechniques 18:130-135.

13.Zammatteo, N., I. Alexandre, I. Ernest, L. Le, F. Brancart and J. Remacle. 1997. Comparison between microwell and bead supports for the detection of human cytomegalovirus amplicons by sandwich hybridization. Anal. Biochem. 253:180-189.

Address correspondence to Dr. Stephen J. Mc Connell, Chugai Biopharmaceuticals, 6275 Nancy Ridge Drive, San Diego, CA 92121, USA. Internet: smcconnell@ chugaibio.com

Received 7 July 1998; accepted 17 September 1998.

Stephen J. McConnell, Thai Dinh, Mong-Huong Le and Dominic G. Spinella Chugai Biopharmaceuticals San Diego, CA, USA 